\title{
Interplay of nasal and rectal carriage of Staphylococcus aureus in intensive care unit patients
}

\author{
Julie Gagnaire ${ }^{1,2}$. Elisabeth Botelho-Nevers ${ }^{1,2}$. Patricia Martin-Simoes ${ }^{3,4}$ - Jérôme Morel ${ }^{5} \cdot$ Fabrice Zéni $^{6}$. \\ Nicolas Maillard ${ }^{7}$. Christophe Mariat ${ }^{7}$. Cyrille H. Haddar ${ }^{1,8}$. Anne Carricajo ${ }^{8}$. Nathalie Fonsale ${ }^{8}$. \\ Florence Grattard $^{1,8}$. Bruno Pozzetto ${ }^{1,8}$. Frédéric Laurent ${ }^{3,4}$. Philippe Berthelot ${ }^{1,2,8} \cdot$ Paul O. Verhoeven ${ }^{1,8}$ (iD
}

Received: 9 April 2019 / Accepted: 11 June 2019 / Published online: 4 July 2019

(C) Springer-Verlag GmbH Germany, part of Springer Nature 2019

\begin{abstract}
The aim of this study was to investigate the relationship between nasal and rectal Staphylococcus aureus carriage in intensive care unit (ICU) patients and the occurrence of ICU-acquired infections related to $S$. aureus carriage. Three hundred and ninety-five patients admitted in ICU were screened for $S$. aureus nasal and rectal carriages and followed to record $S$. aureus infections during their stay. S. aureus strains were genotyped by arbitrarily primed PCR, spatyping, microarray and whole genome sequencing. At ICU admission, 112 of 363 (30.9\%) patients carried S. aureus including $61(16.8 \%)$ exclusive nasal carriers, 40 (11.0\%) combined nasal and rectal carriers and 11 (3.0\%) exclusive rectal carriers. The $152 \mathrm{~S}$. aureus isolates from nasal and rectal swabs belonged to 19 clonal complexes (CCs). Patients colonized in both nose and rectum harboured different strains in at least $40 \%$ of cases according to arbitrarily primed PCR data. Nasal carriers of CC5 S. aureus had an increased risk of rectal carriage $(\mathrm{RR}=1.85$, $P<.05)$. S. aureus nasal and rectal carriage was a risk factor of $S$. aureus ICU-acquired infection $(\mathrm{RR}=4.04 ; 95 \% \mathrm{CI}$ [1.38-11.76]). Incidence rates of endogenous ICU-acquired infections in exclusive nasal carriers, exclusive rectal carriers and in both nasal and rectal carriers were $0.08(5 / 61), 0.09(1 / 11)$ and $0.03(1 / 40)$, respectively $(p=0.47)$. Rectal swabbing increased the detection of $S$. aureus carriage and revealed an important diversity of $S$. aureus strains in ICU patients. Further studies are needed to understand how $S$. aureus rectal carriage increases the risk of endogenous ICU-acquired infections.
\end{abstract}

Keywords Staphylococcus aureus $\cdot$ Colonization $\cdot$ Nose $\cdot$ Rectum $\cdot$ Staphylococcal infections $\cdot$ Intensive care unit

Electronic supplementary material The online version of this article (https://doi.org/10.1007/s10096-019-03613-z) contains supplementary material, which is available to authorized users.

Paul O. Verhoeven

paul.verhoeven@univ-st-etienne.fr

1 GIMAP EA 3064 (Groupe Immunité des Muqueuses et Agents Pathogènes), Université Jean Monnet, Université de Lyon, St-Etienne, France

2 Infectious Diseases Department, University Hospital of St-Etienne, St-Etienne, France

3 CIRI (Centre International de Recherche en Infectiologie), Inserm U1111, Ecole Normale Supérieure de Lyon, Université Lyon 1, CNRS, UMR5308, Université de Lyon, Lyon, France
4 Centre National de Référence des Staphylocoques, Institut des Agents Infectieux, Hospices Civils de Lyon, Lyon, France

5 Department of Anaesthesiology and Intensive Care Medicine, University Hospital of St-Etienne, St-Etienne, France

6 Medical Intensive Care Unit, University Hospital of St-Etienne, St-Etienne, France

7 Nephrology, Dialysis and Renal Transplantation Department, University Hospital of St-Etienne, St-Etienne, France

8 Laboratory of Infectious Agents and Hygiene, University Hospital of St-Etienne, St-Etienne, France 


\section{Introduction}

Staphylococcus aureus colonizes the skin and mucosal membranes of approximately one third of human beings [1-3]. Nasal colonization is a well-established risk factor of different types of $S$. aureus infections in all populations that have been studied [2]. In most cases, the $S$. aureus strains isolated from the nose and the infection site(s) are indistinguishable but the mechanisms of colonization-related infection (i.e. endogenous infection with the strain of carriage) are poorly understood [2]. Still, there is a lack of evidence to define whether $S$. aureus reaches preferentially the site of infection by contamination from the cutaneous site or by translocation through mucosal membrane [4]. The latest data indicate that the mean prevalence of $S$. aureus colonization is $24 \%$ and $14 \%$ in the nose and intestinal tract, respectively $[1,2]$. Compared with the vestibulum nasi, which is the main site of carriage in humans, the gastro-intestinal tract also needs to be considered as an important reservoir of $S$. aureus in humans. However, most studies about $S$. aureus intestinal colonization conducted in the past decades have focussed on methicillin-resistant $S$. aureus (MRSA), and the role of methicillinsusceptible $S$. aureus (MSSA) intestinal carriage in colonization-related infection has been largely neglected. To the best of our knowledge, only a few epidemiological studies comparing nasal and intestinal carriage showed that $S$. aureus intestinal colonization could be an additional risk factor for $S$. aureus infections [5-7]. More recent data showed that a particular clone of S. aureus (i.e. ST228 MRSA) could be linked with lasting colonization of the intestinal tract [8]. Together, these results suggest that the $S$. aureus intestinal reservoir could play a significant role in colonization-related infections and outbreaks. Besides, S. aureus decolonization based on topical application of mupirocine and chlorhexidine for preventing $S$. aureus colonization-related infection did not reduce the intestinal colonization rate [9] and antibiotics-based decolonisation could not be recommended due to the risk of emergence of multidrugresistant bacteria. Thus, prevention strategies taking into account intestinal $S$. aureus reservoir could help reduce colonization-related $S$. aureus infections, especially in surgical or critically-ill patients.

In order to better understand the relationship between $S$. aureus nasal and intestinal colonization whatever the susceptibility to methicillin, we conducted a prospective study in a cohort of ICU patients that aimed to investigate (i) the prevalence of $S$. aureus nasal and intestinal colonization, (ii) the clonality of $S$. aureus strains isolated in both sites and (iii) the risk of colonization-related infections during hospitalization in ICU.

\section{Material and methods}

Patient and sample collection The study took place at the University Hospital of St-Etienne, France, between February and July 2013 in the three adults ICUs including a nephrological unit of 6 beds, a polyvalent medical and surgical unit of 12 beds and one emergency medical unit of 15 beds. Patients were enrolled prospectively and sampled at admission in ICU using nasal and rectal swabs (eSwab $480 \mathrm{CE}$, Copan, Brescia, Italy). S. aureus infections were collected prospectively by the intensive care physicians during the patients' ICU stays. ICU-acquired infections were defined according to the surveillance definition of health care-associated infection in acute care settings elaborated by Centres for disease control and the National Healthcare Safety Network standards [10]. All medical records of infected patients were reviewed independently by both an infectious disease (EBN) and an infection control (JG) specialists. S. aureus infection was defined as ICU-acquired if it occurred more than $48 \mathrm{~h}$ after patient's admission in the ICU. Acquired-infections were considered endogenous if the strain responsible for infection shared both the same spa-type and the same microarray profile as the one(s) found in nasal and/or rectal swabs sampled before the occurrence of infection.

Microbiological methods Nasal and rectal swabs were plated on S. aureus chromogenic agar (BBL CHROMAgar Staph aureus, Becton Dickinson, France) by using the EasySpiral Dilute instrument (Interscience, St-Nom-la-Bretèche, France) and incubated at $36{ }^{\circ} \mathrm{C}$ for $48 \mathrm{~h}$. The Scan 1200 plate reader (Interscience) was used to quantify $S$. aureus loads [11]. Clinical specimens were plated on blood agar (COS ref.: 43049 , bioMérieux, France) and incubated at $36^{\circ} \mathrm{C}$ for at least $48 \mathrm{~h}$. All presumptive colonies were identified by MALDITOF MS according manufacturer recommendations (Microflex LT, Bruker, Bremen, Germany).

Genotyping of S. aureus strains $S$. aureus strains were analysed by arbitrarily-primed PCR [12], spa-typing [13] and DNA microarray ( $S$. aureus Genotyping Kit v2.0, Alere, France) [14]. Clonal complexes (CC) of $S$. aureus strains were assigned by using the results of DNA microarray [14]. Microarray data and spa-types were analysed with BioNumerics software v7.6 (Applied Maths, Sint-MartensLatem, Belgium). To analyse more closely the relationship between strains isolated from two patients colonized at both nasal and rectal sites and who presented $S$. aureus infection, Illumina 300 bp paired-end whole genome shotgun sequencing was performed as previously described [15]. Core genome single-nucleotide polymorphism (SNP) analysis and pairwise distance matrices were produced with Snippy v4.2.1 (https:// github.com/tseemann/snippy), by using the rectal strain sequence as reference. S. aureus strains isolated from within 
a single individual were considered as unrelated when pairwise SNPs were greater than 40 [16].

Statistical methods Statistical analysis was performed using SPSS software (IBM SPSS v20.0, Chicago, IL, USA) and MedCalc statistical software v18.11 (MedCalc Software bvba, Ostend, Belgium). Chi-squared and Fisher's exact tests were used to compare categorical variables. Mann-Whitney $U$ test was used to compare continuous variables. $p$ values below 0.05 were considered as statistically significant. Minimum spanning tree was computed with BioNumerics v7.6 (Applied Maths, Sint-Martens-Latem, Belgium).

Ethical statement This study was approved by an Institutional Review Board ("Comité de Protection des Personnes Sud-Est I" No 2012-28) and by the National Security Agency of Medicines and Health Products.

\section{Results}

Patient population From the 400 patients enrolled, 363 were analysed in this study (Fig. 1). The sex ratio (M/F) was 1.6; the mean $( \pm \mathrm{SD})$ age was $61 \pm 16$ years and the median (range) of Simplified Acute Physiologic Score II was 38 (6-115).

S. aureus nasal and rectal carriage At ICU admission, 112 of 363 (30.9\%) patients carried S. aureus: 61 (54.5\%) were exclusive nasal carriers including 6 MRSA carriers, 40 (35.7\%) were co-carriers (i.e. carriers of $S$. aureus in both nose and rectum) including 2 MRSA carriers, and 11 (9.8\%) were exclusive rectal carriers. Thus, rectal swab improved the detection of $S$. aureus carriage by $10 \%$ (11/112). The characteristics of the studied population, according to $S$. aureus carriage status, are described in Table 1. Except for the risk of acquired infection (see below), characteristics of patients were similar between $S$. aureus carriers and non-carriers. Notably, exposure to antimicrobials with activity against $S$. aureus was similar between $S$. aureus carriers and non-carriers (Table 1).

At inclusion, the mean $( \pm \mathrm{SD}) S$. aureus load was significantly higher in nasal specimens $(5.1 \pm 2.2 \log$ CFU/swab) than in rectal specimens $(3.3 \pm 1.3 \log \mathrm{CFU} / \mathrm{swab})$ $(p<0.0001)$ (Fig. 2a). The mean $( \pm \mathrm{SD}) S$. aureus nasal loads were $5.6 \pm 2.1$ and $4.7 \pm 2.2 \log \mathrm{CFU} / \mathrm{swab}$ in co-carriers and in sole nasal carriers, respectively ( $p=0.053$ ) (Fig. 2b). The mean $( \pm \mathrm{SD}) S$. aureus rectal loads were $3.4 \pm 1.3$ and $3.1 \pm$ 1.4 CFU/swab in co-carriers and in sole rectal carriers, respectively ( $p=0.6)$ (Fig. 2c).

Genetic diversity of S. aureus strains The 152 S. aureus isolates recovered from nasal and rectal swabs belonged to 20 clonal complexes (CCs). Sixteen (10.5\%) isolates were meticillin-resistant including 14 isolates belonging to CC8 (Lyon clone, EMRSA-2) and two isolates belonging to CC22 (EMRSA-15) (Fig. 3).

Arbitrarily primed PCR showed that strains recovered from patients with combined nasal and rectal $S$. aureus carriages are different between the two sites in 16 patients out of $40(40 \%)$. In other words, the rectal sampling improved the recovery of $S$. aureus strains colonizing humans up to $24 \%(27 / 112)$.

Interestingly, nasal carriers of $S$. aureus strains belonging to CC5 have been found to have an increased risk of $S$. aureus rectal carriage $(\mathrm{RR}=1.85,95 \% \mathrm{CI}[1.14-3.02])$. No other
Fig. 1 Flowchart: study design and distribution of the studied population in the three adult ICUs of the University hospital of StEtienne, France

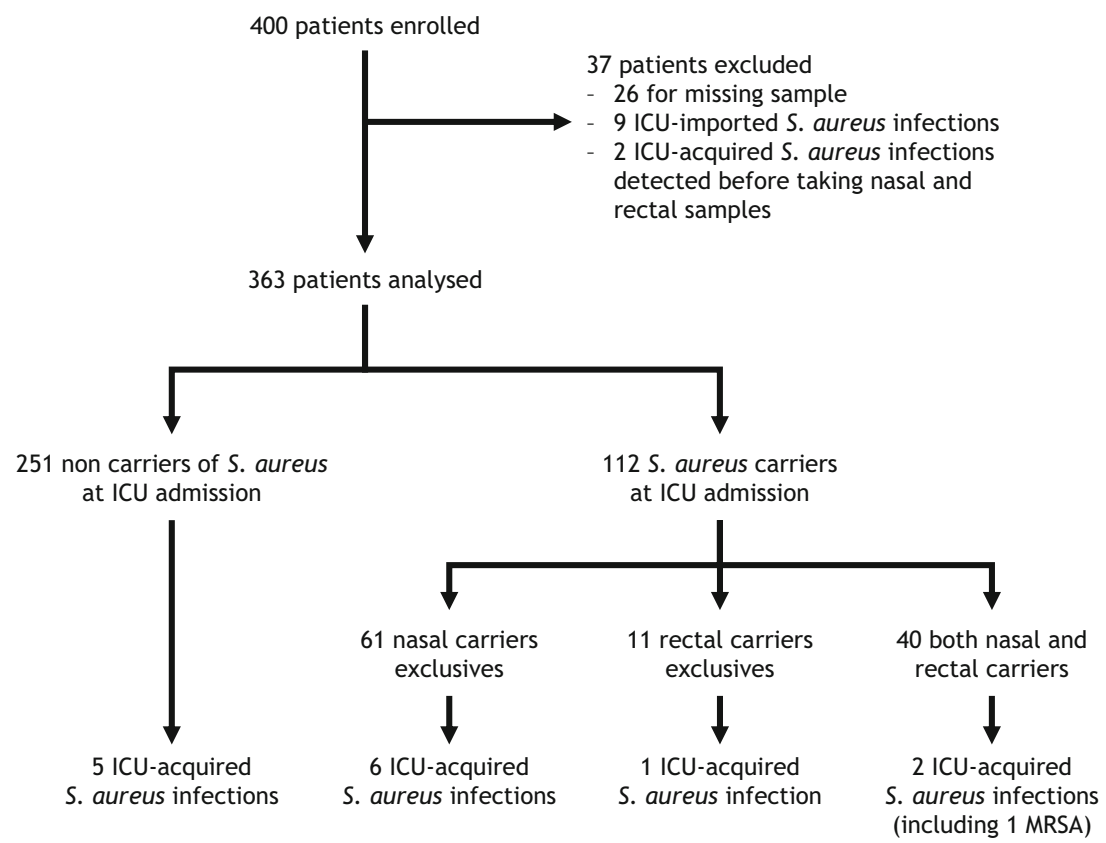


Table 1 Characteristics of patients admitted in ICUs according to their $S$. aureus carrier state

\begin{tabular}{llll}
\hline & $\begin{array}{l}\text { S. aureus carriers } \\
\text { at ICU admission } \\
n=112(\%)\end{array}$ & $\begin{array}{l}\text { Non } S \text {. aureus carriers } \\
\text { at ICU admission } \\
n=251(\%)\end{array}$ & $P$ value \\
\hline Type of ICU & & & \\
Medical and surgical & $55(49.1)$ & $111(44.2)$ & $\mathrm{ns}$ \\
Emergency medical & $38(33.9)$ & $81(32.3)$ & $\mathrm{ns}$ \\
Nephrological & $19(17.0)$ & $59(23.5)$ & $\mathrm{ns}$ \\
Sex ratio (M/F) & 1.8 & 1.5 & $\mathrm{~ns}$ \\
Age (mean \pm SD) & $60 \pm 17$ & $62 \pm 15$ & $\mathrm{~ns}$ \\
SAPSII ${ }^{\text {a }}$ (mean \pm SD) & $42 \pm 22$ & $41 \pm 20$ & $\mathrm{~ns}$ \\
S. aureus acquired infections & $9(8.0)$ & $5(2.0)$ & $<0.05$ \\
Bacteremia & $6(5.4)$ & $1(0.4)$ & $\mathrm{n} 0.01$ \\
Pneumonia (incl. VAP) & $3(2.7)$ & $4(1.6)$ & $\mathrm{ns}$ \\
Antimicrobial treatment with activity against & $45(40.2)$ & $112(44.6)$ & \\
$\quad$ S. aureus before nasal and rectal samplings & & & \\
\hline
\end{tabular}

${ }^{\text {a }}$ Simplified acute physiologic score II

${ }^{\mathrm{b}}$ Including four cases of catheter-related bacteremia in carrier group and one case non-carrier group

$V A P$ ventilator-associated pneumonia clonal complex was found to influence the risk of associated rectal carriage (Table 2).

\section{S. aureus acquired infections Fourteen ICU-acquired}

$S$. aureus infections, including one due to MRSA, were diagnosed during the study period and are summarized in Table 3. Nine $S$. aureus infections occurred in carriers and 5 in noncarriers. S. aureus carriage was significantly associated with the occurrence of $S$. aureus ICU-acquired infections (RR = 4.30; 95\% CI [1.41-13.14]). Overall, the density incidence of ICU-acquired $S$. aureus infections was 2.2 per $1000 \mathrm{ICU}$ days, respectively, 4.66 and 1.1 per 1000 ICU-days in $S$. aureus carriers and non-carriers $(p<0.01)$. The median (interquartile range) of infection onset after ICU admission was 16 (11 to 19$)$ and 12 days (3.75 to 27.5 ) in carriers and noncarriers, respectively $(p=0.39)$.

$S$. aureus infections occurring in carriers were due to their endogenous strain in 8 out of 9 cases (Table 3 ). Incidence rates of endogenous infections were, respectively, 0.08 (5/61), 0.09 $(1 / 11)$ and $0.03(1 / 40)$ in exclusive nasal carriers, exclusive rectal carriers and in both nasal and rectal carriers $(p=0.47)$. One patient (case no. 6) acquired a $S$. aureus bloodstream infection related to a femoral arterial-catheter and had a previous rectal colonization with high bacterial load $\left(10^{5} \mathrm{CFU} /\right.$ swab) and no $S$. aureus nasal colonization (Table 3). Two patients (cases number 9 and 10) were found to carry $S$. aureus in the nose and in the rectum and acquired S. aureus after ICU admission (Table 3). Briefly, in case no. 8, S. aureus isolates recovered from the nose and the rectum harboured similar DNA microarray profiles and as little as 6 SNPs (>97\% reference genome mapped). On the one hand, the strain isolated in blood culture showed a deletion of 2 spa repeats compared to the nasal and rectal isolates using spatyping method. On the other hand, whole genome sequencing showed 894 and 896 pairwise SNPs compared to the nasal and rectal isolates respectively. In case no. 9, S. aureus isolates
Fig. 2 S. aureus loads recovered from nasal and rectal specimens of the 363 patients included in the study (a), from nasal specimens of co-carriers and nasal carriers sole (b) and from rectal specimens of co-carriers and rectal carrier sole (c)

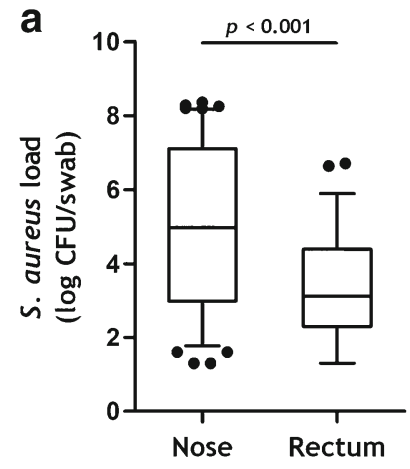

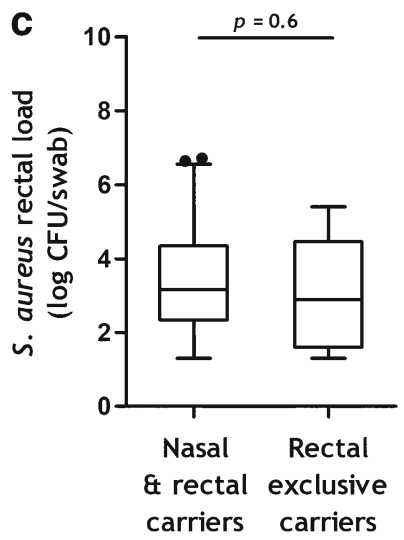




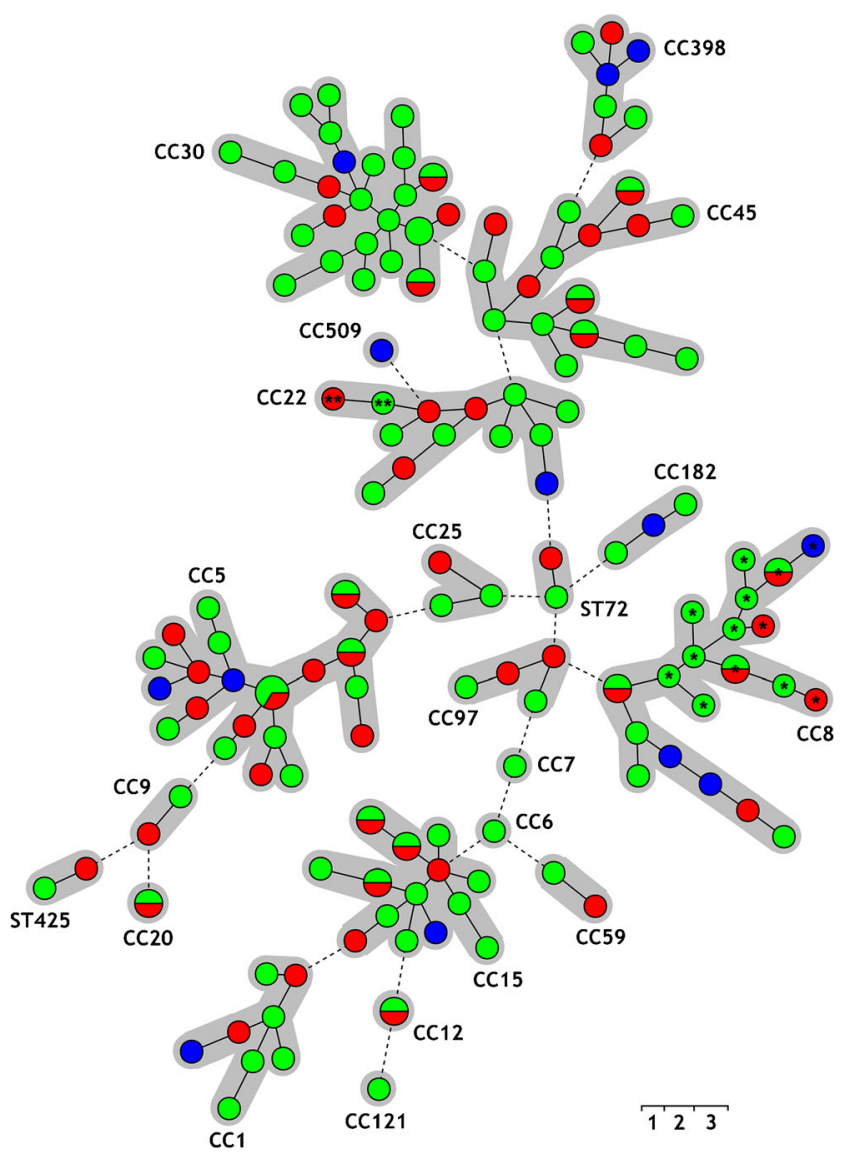

Fig. 3 Minimum spanning tree based on DNA microarray analysis of 165 $S$. aureus isolates recovered from nose (green), rectum (red) and infection site (blue) in intensive care unit patients. Clonal complexes (CC) or sequence type (ST) assigned by microarray analysis are highlighted in light grey. The node size represents the number of isolates according to the scale given. *CC8-MRSA-IV [sea+], Lyon Clone/UK-EMRSA-2. **CC22-MRSA-IV [fnbB-,sec/l-], UK-EMRSA-15/Barnim EMRSA

grown from the nasal swabs, rectal swabs and blood culture shared the same spa-type and similar DNA microarray profiles, and harboured no more than 9 pairwise SNPs (Table 3).

\section{Discussion}

In ICU patients, we found a prevalence of nasal (28\%) and rectal $(14 \%)$ carriages very similar to other settings $[1,2]$ despite frequent exposure to antimicrobial drugs with activity against $S$. aureus in this population. Exclusive rectal carriage occurred rarely in our cohort (3\%), which confirms the results found in other populations [1, 17]. Although nasal carriage was more frequent than rectal carriage, we found that the incidence of endogenous infection in exclusive rectal carriers is similar to those observed in exclusive nasal carriers. To date, few is known about the risk of infection related to colonization at other sites than the nose. In this prospective cohort, we reported a well-documented case of endogenous infection in a patient with exclusive rectal carriage (see case 6; Table 3) and 2 other cases of endogenous $S$. aureus infections occurring in patients colonized at both sites (cases 8 and 9; see Table 3). Previously, two studies reported three cases of $S$. aureus infections in $S$. aureus exclusive rectal carriers. However, molecular typing of colonization and infection strains was not performed and the time between colonization and the occurrence of infection was not specified $[6,18]$. Additionally, Szumowski et al. showed that MRSA perianal colonization was associated with skin and soft tissue infection (SSTI) but they did not compare perianal and SSTI isolates [7]. Squier et al. reported that patients with both rectal and nasal carriage were significantly more likely to develop $S$. aureus infection than those with nasal carriage only in ICU but they failed to find infection in exclusive rectal carriers [5]. Here, in the case of bacteremia occurring in an exclusive rectal carrier, it can be hypothesized that the catheter proximity to the colonized site and the high bacterial load in the rectum led to the occurrence of a bloodstream infection. For the two other cases, since strains of colonization were similar, it is impossible to know which site of colonization was at the origin of the infection. In case 8, the strain isolated from the blood culture was considered as different from those
Table 2 Risk of $S$. aureus rectal carriage in ICU patients according to the clonal complex of the $S$. aureus nasal carriage strain

\begin{tabular}{llll}
\hline $\begin{array}{l}\text { Clonal complex of } S \text {. aureus } \\
\text { nasal isolate (no. of patients) }\end{array}$ & Relative risk of rectal carriage & $95 \%$ confidence interval & $P$ value \\
\hline CC30 (21) & 1.11 & $0.63-1.95$ & $\mathrm{~ns}$ \\
CC8 (15) & 0.82 & $0.38-1.75$ & $\mathrm{~ns}$ \\
CC5 (12) & 1.85 & $1.14-3.02$ & $<0.05$ \\
CC45 (12) & 1.31 & $0.70-2.44$ & $\mathrm{~ns}$ \\
CC15 (11) & 0.87 & $0.38-1.96$ & $\mathrm{~ns}$ \\
CC22 (8) & 0.94 & $0.37-2.38$ & $\mathrm{~ns}$ \\
CC398 (3) & 0.31 & $0.02-4.13$ & $\mathrm{~ns}$ \\
CC1 (5) & 0.49 & $0.08-2.89$ & $\mathrm{~ns}$ \\
CC97 (2) & 1.27 & $0.31-5.18$ & $\mathrm{~ns}$ \\
Others CCs (12) & 0.60 & $0.22-1.65$ & $\mathrm{~ns}$ \\
\hline
\end{tabular}




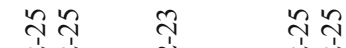

สิ่

के के कृत के के

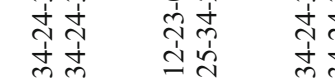

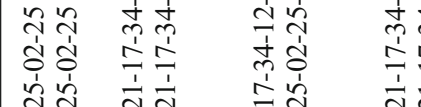

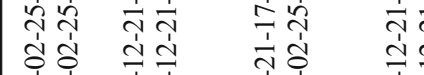

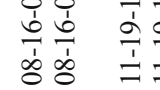

总号

तิ

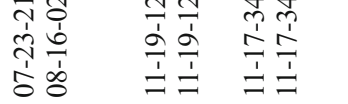

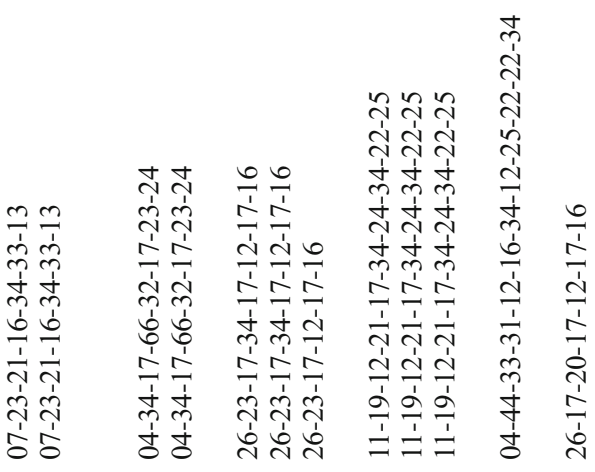

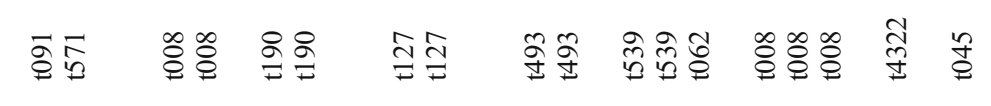

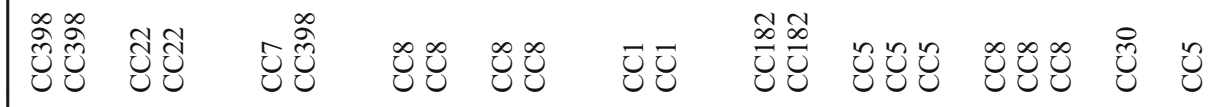

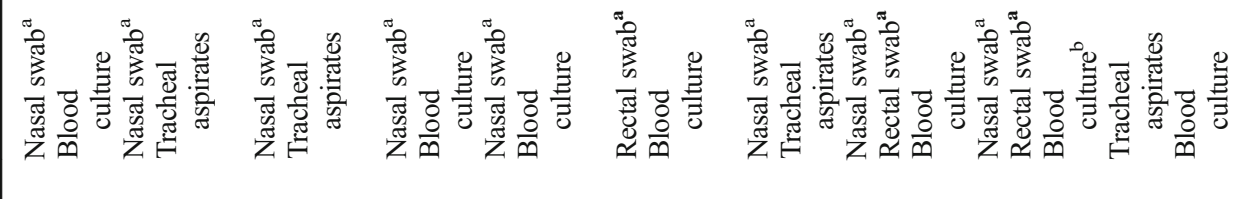

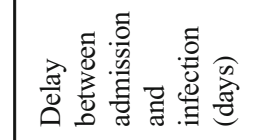

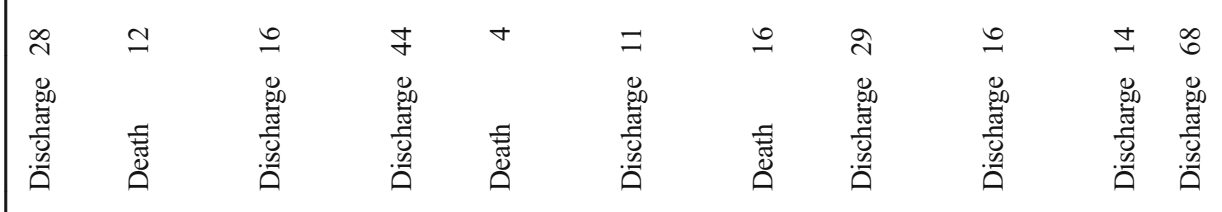

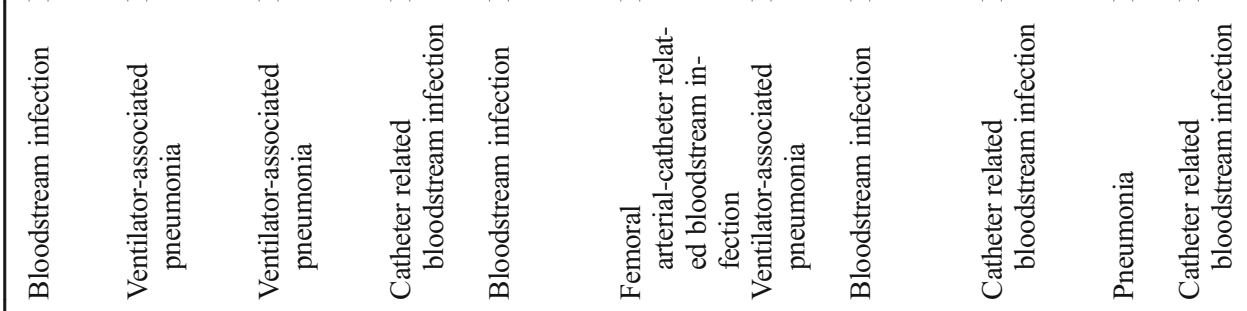

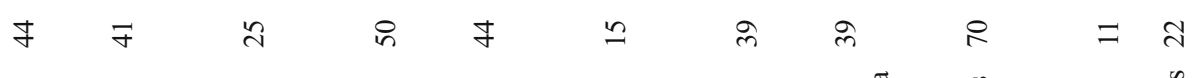

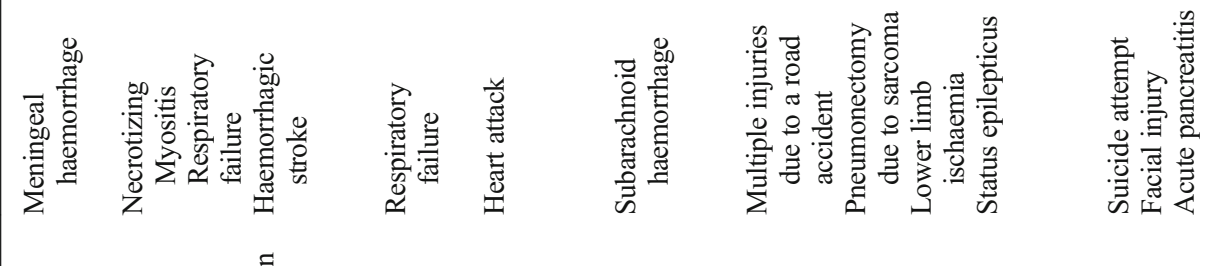

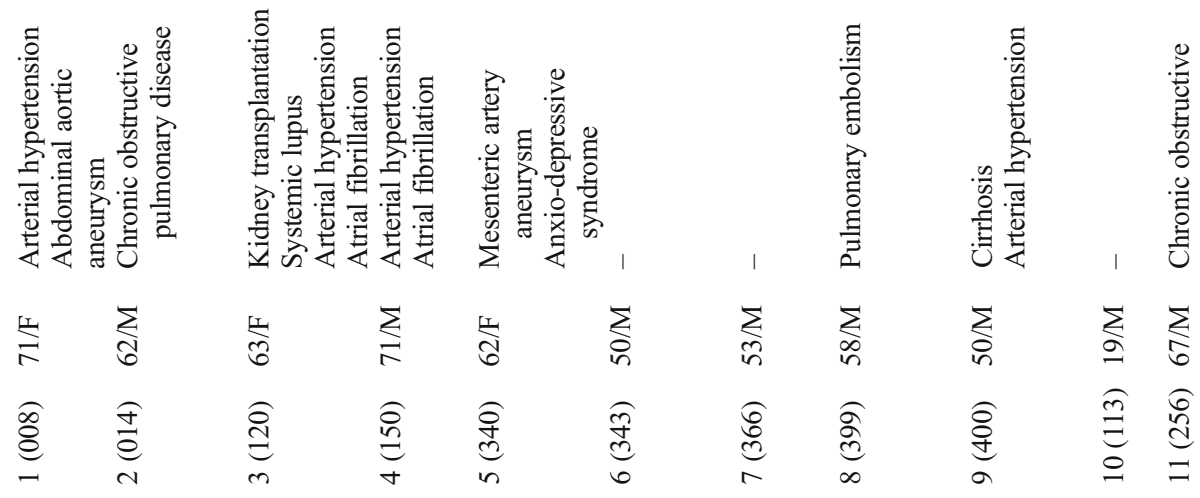


recovered from the nose and the rectum of this patient given the large number of pairwise SNPs observed. In this case, the delay of infection occurred 29 days after ICU admission, which could be enough to let the bacteria adapt to the host [19]. In case 9, WGS sequencing showed that the strain isolated in blood culture and those isolated from nasal and rectal sites were closely related, and despite the use of highthroughput method for investigation [20], it was not possible to determine whether nasal or rectal colonization originated the infection. Both cases of bacteremia might be related to rectal carriage. As we observed here, the combination of colonization sites is also associated to a high $S$. aureus bacterial load and then could potentially facilitate staphylococcal infections, increasing the risk of device contamination [18]. These observations evoke that the rectal reservoir could play a role in autoinfection [21]. The combination of colonization sites is probably associated to a dispersion of $S$. aureus colonization on the body (skin and mucosa) and in the environment of the patient [18]. Additionally, Senn et al., who reported a large outbreak of $S$. aureus colonization, suggested that the $S$. aureus rectal strain plays the role of a stealthy superbug able to maintain a long-term hospital outbreak by crosstransmission [8]. In our work, the genotyping of the 165 $S$. aureus isolates revealed a great diversity within patients indicating that no or few cross-transmissions occurred in the ICUs. Interestingly, we showed that strains belonging to CC5 seem to be more implicated in rectal carriage, notably when the patient was co-colonized with this clone in nose and rectum compared to other clones. The ST228 was already related to rectal carriage in an outbreak of $S$. aureus colonization [8].

Staphylococcus aureus is one of the most frequent causes of infections in the ICU [22-24] and we confirmed that $S$. aureus carriage is a risk factor for $S$. aureus ICU-acquired infections. This risk factor had been largely described previously for MRSA carriage [22, 25-29] but not so extensively for MSSA carriage [30, 31]. In recent studies [22, 25-30, 32], the relative risk of acquisition of $S$. aureus infections in ICU associated with $S$. aureus nasal or throat carriage ranged from 2.5 to 17.8 and our data are in concordance to previous findings. In fact recently, studies showed that screening patients for MRSA carriage at ICU admission is a valuable tool for guiding decision to treat ICU-acquired infections [27, 28]. We highlighted in the present work that not only MRSA carriage but also MSSA carriage could have an impact on the risk of infection in ICU as also showed by Paling et al. in a post hoc analysis of two cohort studies in critically ill patients [32]. Interestingly, molecular typing of $S$. aureus strains in ICUpatients harbouring combined nasal and rectal carriage showed that approximately half of them were colonized with two different strains of $S$. aureus. Similarly, we reported that $S$. aureus naso-pharyngeal carriers harboured two different strains in approximately half of cases in a cohort of haemodialysis patients [33]. These results suggest that 
S. aureus carriers could harbour two or three different strains in the nose, the throat and the rectum in hospitalized patients exposed to colonization pressure and antimicrobial drugs [34]. Therefore, screening S. aureus carriage at ICU admission, for whatever methicillin resistance profile, would allow physicians, especially among patients with pneumonia, to adapt a probabilistic antibiotic treatment for patients according to the antimicrobial drug susceptibility of their $S$. aureus carriage strain with potential antimicrobial stewardship implications [35]. The prevention of S. aureus (MSSA and MRSA) endogenous infections may also be questioned in further studies since a recent meta-analysis showed that mupirocin decolonization was protective against $S$. aureus infections among adult ICU patients [36].

There are some limitations to our study. First, patients have been enrolled in 2013 and the study data may not represent the present situation. In the present work, we did not investigate other reservoirs as the study was observational without additional sampling of the patients. Additionally, because of the high rate of patients exposed to antimicrobial drugs, the prevalence of $S$. aureus carriage was probably underestimated and not reliable to determine the $S$. aureus nasal carrier status, as described by our team $[37,38]$ when we investigated the relationship between persistent and non-persistent carriers and the risk of developing a ICU-acquired $S$. aureus infection. However, this study is relevant in a population of ICU patients because of the frequent prescription of antibiotics in all ICUs.

\section{Conclusion}

This study confirms that nasal colonization by $S$. aureus is an important risk factor for $S$. aureus ICU-acquired infections and suggests that $S$. aureus rectal carriage could be an additional risk factor for infection. Rectal swabbing could improve the detection of an unrecognized S. aureus strain. Screening of S. aureus carriage in ICU patients, for whatever methicillin resistance profile, may help the prescription of antibiotics. Further studies are needed to better understand the role of the different reservoirs of $S$. aureus strains and their clinical impact on the risk of $S$. aureus infection, notably in ICUs.

Acknowledgments The investigators thank the nursing and medical staff of the adults ICU of the University Hospital of St-Etienne involved in the sampling collection and in the collection of data. Amélie Epercieux, Laurie Sahuc, Marine Servant, Laura Dimier and the staff of laboratory of Infectious Agents and Hygiene of the University Hospital of St-Etienne are acknowledged for their skilful technical assistance.

Funding This research has received funding support from the University Hospital of St-Etienne (2012-A01182-41) and was promoted by the University Hospital of St-Etienne, France.

\section{Compliance with ethical standards}

Conflict of interest The authors declare that they have no conflicts of interest.

\section{References}

1. Gagnaire J, Verhoeven PO, Grattard F, Rigaill J, Lucht F, Pozzetto B et al (2017) Epidemiology and clinical relevance of Staphylococcus aureus intestinal carriage: a systematic review and meta-analysis. Expert Rev Anti-Infect Ther 15:767-785. https:// doi.org/10.1080/14787210.2017.1358611

2. Verhoeven PO, Gagnaire J, Botelho-Nevers E, Grattard F, Carricajo A, Lucht F et al (2014) Detection and clinical relevance of Staphylococcus aureus nasal carriage: an update. Expert Rev Anti-Infect Ther 12:75-89. https://doi.org/10.1586/14787210. 2014.859985

3. Wertheim HFL, Melles DC, Vos MC, van Leeuwen W, van Belkum A, Verbrugh HA et al (2005) The role of nasal carriage in Staphylococcus aureus infections. Lancet Infect Dis 5:751-762. https://doi.org/10.1016/S1473-3099(05)70295-4

4. Krezalek MA, Hyoju S, Zaborin A, Okafor E, Chandrasekar L, Bindokas V et al (2017) Can methicillin-resistant Staphylococcus aureus silently travel from the gut to the wound and cause postoperative infection? Modeling the "Trojan horse hypothesis.". Ann Surg 267:749-758. https://doi.org/10.1097/SLA. 0000000000002173

5. Squier C, Rihs JD, Risa KJ, Sagnimeni A, Wagener MM, Stout J et al (2002) Staphylococcus aureus rectal carriage and its association with infections in patients in a surgical intensive care unit and a liver transplant unit. Infect Control Hosp Epidemiol 23:495-501. https://doi.org/10.1086/502095

6. Srinivasan A, Seifried SE, Zhu L, Srivastava DK, Perkins R, Shenep JL et al (2010) Increasing prevalence of nasal and rectal colonization with methicillin-resistant Staphylococcus aureus in children with cancer. Pediatr Blood Cancer 55:1317-1322. https:// doi.org/10.1002/pbc. 22815

7. Szumowski JD, Wener KM, Gold HS, Wong M, Venkataraman L, Runde CA et al (2009) Methicillin-resistant Staphylococcus aureus colonization, behavioral risk factors, and skin and soft-tissue infection at an ambulatory clinic serving a large population of HIVinfected men who have sex with men. Clin Infect Dis 49:118 121. https://doi.org/10.1086/599608

8. Senn L, Clerc O, Zanetti G, Basset P, Prod'hom G, Gordon NC et al (2016) The stealthy superbug: the role of asymptomatic enteric carriage in maintaining a long-term hospital outbreak of ST228 methicillin-resistant Staphylococcus aureus. MBio 7:e02039e02015. https://doi.org/10.1128/mBio.02039-15

9. Wertheim HFL, Verveer J, Boelens HAM, van Belkum A, Verbrugh HA, Vos MC (2005) Effect of mupirocin treatment on nasal, pharyngeal, and perineal carriage of Staphylococcus aureus in healthy adults. Antimicrob Agents Chemother 49:1465-1467. https://doi.org/10.1128/AAC.49.4.1465-1467.2005

10. Horan TC, Andrus M, Dudeck MA (2008) CDC/NHSN surveillance definition of health care-associated infection and criteria for specific types of infections in the acute care setting. Am J Infect Control 36:309-332. https://doi.org/10.1016/j.ajic.2008.03.002

11. Verhoeven PO, Noyel P, Bonneau J, Carricajo A, Fonsale N, Ros A et al (2014) Evaluation of the new brilliance GBS chromogenic medium for screening of Streptococcus agalactiae vaginal colonization in pregnant women. J Clin Microbiol 52:991-993. https:// doi.org/10.1128/JCM.02926-13 
12. Verhoeven P, Grattard F, Carricajo A, Pozzetto B, Berthelot P (2010) Better detection of Staphylococcus aureus nasal carriage by use of nylon flocked swabs. J Clin Microbiol 48:4242-4244. https://doi.org/10.1128/JCM.01425-10

13. Harmsen D, Claus H, Witte W, Rothgänger J, Claus H, Turnwald D et al (2003) Typing of methicillin-resistant Staphylococcus aureus in a university hospital setting by using novel software for spa repeat determination and database management. J Clin Microbiol 41:5442-5448. https://doi.org/10.1128/JCM.41.12.5442-5448. 2003

14. Monecke S, Slickers P, Ehricht R (2008) Assignment of Staphylococcus aureus isolates to clonal complexes based on microarray analysis and pattern recognition. FEMS Immunol Med Microbiol 53:237-251. https://doi.org/10.1111/j.1574-695X.2008. 00426.x

15. Durand G, Javerliat F, Bes M, Veyrieras J-B, Guigon G, Mugnier N et al (2018) Routine whole-genome sequencing for outbreak investigations of Staphylococcus aureus in a national reference center. Front Microbiol 9:511. https://doi.org/10.3389/fmicb.2018.00511

16. Golubchik T, Batty EM, Miller RR, Farr H, Young BC, LarnerSvensson H et al (2013) Within-host evolution of Staphylococcus aureus during asymptomatic carriage. PLoS One 8:e61319. https:// doi.org/10.1371/journal.pone.0061319

17. Acton DS, Plat-Sinnige MJT, van Wamel W, de Groot N, van Belkum A (2009) Intestinal carriage of Staphylococcus aureus: how does its frequency compare with that of nasal carriage and what is its clinical impact? Eur J Clin Microbiol Infect Dis 28: 115-127. https://doi.org/10.1007/s10096-008-0602-7

18. Bhalla A, Aron DC, Donskey CJ (2007) Staphylococcus aureus intestinal colonization is associated with increased frequency of S. aureus on skin of hospitalized patients. BMC Infect Dis 7:105. https://doi.org/10.1186/1471-2334-7-105

19. Benoit JB, Frank DN, Bessesen MT (2018) Genomic evolution of Staphylococcus aureus isolates colonizing the nares and progressing to bacteremia. PLoS One 13:e0195860. https://doi. org/10.1371/journal.pone.0195860

20. Diep BA (2013) Use of whole-genome sequencing for outbreak investigations. Lancet Infect Dis 13:99-101. https://doi.org/10. 1016/S1473-3099(12)70276-1

21. van Belkum A (2016) Hidden Staphylococcus aureus carriage: overrated or underappreciated? MBio 7:e00079-e00016. https:// doi.org/10.1128/mBio.00079-16

22. Kao KC, Chen CB, Hu HC, Chang HC, Huang CC, Huang YC (2015) Risk factors of methicillin-resistant Staphylococcus aureus infection and correlation with nasal colonization based on molecular genotyping in medical intensive care units: a prospective observational study. Medicine (Baltimore) 94:e1100. https://doi.org/10. 1097/MD.0000000000001100

23. Vincent JL, Rello J, Marshall J, Silva E, Anzueto A, Martin CD et al (2009) International study of the prevalence and outcomes of infection in intensive care units. JAMA 302:2323-2329. https://doi.org/ 10.1001/jama.2009.1754

24. Weber DJ, Raasch R, Rutala WA (1999) Nosocomial infections in the ICU: the growing importance of antibiotic-resistant pathogens. Chest 115:34S-41S. https://doi.org/10.1378/chest.115.suppl 1.34s

25. Altinbas A, Shorbagi A, Ascioglu S, Zarakolu P, Cetinkaya-Sardan Y (2013) Risk factors for intensive care unit acquired nasal colonization of MRSA and its impact on MRSA infection. J Clin Lab Anal 27:412-417. https://doi.org/10.1002/jcla.21620

26. Jang HC, Choi OJ, Kim GS, Jang MO, Kang SJ, Jung SI et al (2014) Active surveillance of the trachea or throat for MRSA is more sensitive than nasal surveillance and a better predictor of MRSA infections among patients in intensive care. PLoS One 9: e99192. https://doi.org/10.1371/journal.pone.0099192
27. Langsjoen J, Brady C, Obenauf E, Kellie S (2014) Nasal screening is useful in excluding methicillin-resistant Staphylococcus aureus in ventilator-associated pneumonia. Am J Infect Control 42:1014 1015. https://doi.org/10.1016/j.ajic.2014.05.026

28. Tilahun B, Faust AC, McCorstin P, Ortegon A (2015) Nasal colonization and lower respiratory tract infections with methicillinresistant Staphylococcus aureus. Am J Crit Care 24:8-12. https:// doi.org/10.4037/ajcc2015102

29. Ziakas PD, Anagnostou T, Mylonakis E (2014) The prevalence and significance of methicillin-resistant Staphylococcus aureus colonization at admission in the general ICU setting: a meta-analysis of published studies. Crit Care Med 42:433-444. https://doi.org/10. 1097/CCM.0b013e3182a66bb8

30. Keene A, Vavagiakis P, Lee MH, Finnerty K, Nicolls D, Cespedes $\mathrm{C}$ et al (2005) Staphylococcus aureus colonization and the risk of infection in critically ill patients. Infect Control Hosp Epidemiol 26: 622-628. https://doi.org/10.1086/502591

31. Niven DJ, Laupland KB, Gregson DB, Church DL (2009) Epidemiology of Staphylococcus aureus nasal colonization and influence on outcome in the critically ill. J Crit Care 24:583-589. https://doi.org/10.1016/j.jcrc.2008.10.004

32. Paling FP, Wolkewitz M, Bode LGM, Klein Klouwenberg PMC, Ong DSY, Depuydt P et al (2017) Staphylococcus aureus colonization at ICU admission as a risk factor for developing S. aureus ICU pneumonia. Clin Microbiol Infect 23:49.e9-49.e14. https://doi.org/ 10.1016/j.cmi.2016.09.022

33. Verhoeven PO, Haddar CH, Grattard F, Carricajo A, BotelhoNevers E, Pozzetto B et al (2015) Does pharyngeal sampling improve the detection of nasopharyngeal persistent carriers of Staphylococcus aureus? J Inf Secur 70:549-552. https://doi.org/ 10.1016/j.jinf.2015.01.003

34. Batra R, Eziefula AC, Wyncoll D, Edgeworth J (2008) Throat and rectal swabs may have an important role in MRSA screening of critically ill patients. Intensive Care Med 34:1703-1706. https:// doi.org/10.1007/s00134-008-1153-1

35. Brusselaers N, Vogelaers D, Blot S (2011) The rising problem of antimicrobial resistance in the intensive care unit. Ann Intensive Care 1:47. https://doi.org/10.1186/2110-5820-1-47

36. Nair R, Perencevich EN, Blevins AE, Goto M, Nelson RE, Schweizer ML (2016) Clinical effectiveness of mupirocin for preventing Staphylococcus aureus infections in nonsurgical settings: a meta-analysis. Clin Infect Dis 62:618-630. https://doi.org/ $10.1093 / \mathrm{cid} / \mathrm{civ} 901$

37. Verhoeven PO, Gagnaire J, Haddar CH, Grattard F, Thibaudin D, Afiani A et al (2016) Identifying hemodialysis patients with the highest risk of Staphylococcus aureus endogenous infection through a simple nasal sampling algorithm. Medicine (Baltimore) 95:e3231. https://doi.org/10.1097/MD.0000000000003231

38. Verhoeven PO, Grattard F, Carricajo A, Lucht F, Cazorla C, Garraud $\mathrm{O}$ et al (2012) An algorithm based on one or two nasal samples is accurate to identify persistent nasal carriers of Staphylococcus aureus. Clin Microbiol Infect 18:551-557. https:// doi.org/10.1111/j.1469-0691.2011.03611.x

Publisher's note Springer Nature remains neutral with regard to jurisdictional claims in published maps and institutional affiliations. 